\title{
SIMULATION OF LASER-COMPTON COOLING OF ELECTRON BEAMS*
}

\author{
TOMOMI OHGAKI \\ Lawrence Berkeley National Laboratory \\ Berkeley, California 94720, USA
}

\begin{abstract}
We study a method of laser-Compton cooling of electron beams. Using a Monte Carlo code, we evaluate the effects of the laser-electron interaction for transverse cooling. The optics with and without chromatic correction for the cooling are examined. The laserCompton cooling for JLC/NLC at $E_{0}=2 \mathrm{GeV}$ is considered.
\end{abstract}

\section{Introduction}

A novel method of electron beam cooling for future linear colliders was proposed by V.Telnov. 1 During head-on collisions with laser photons, the transverse distribution of electron beams remains almost unchanged and also the angular spread is almost constant. Because the Compton scattered photons follow the initial electron trajectory with a small additional spread due to much lower energy of photons (a few $\mathrm{eV}$ ) than the energy of electrons (several $\mathrm{GeV}$ ). The emittance $\epsilon_{i}=\sigma_{i} \sigma_{i}^{\prime}$ remains almost unchanged $(i=x, y)$. At the same time, the electron energy decreases from $E_{0}$ to $E_{f}$. Thus the normalized emittances have decreased as follows

$$
\epsilon_{n}=\gamma \epsilon=\epsilon_{n 0}\left(E_{f} / E_{0}\right)=\epsilon_{n 0} / C
$$

where $\epsilon_{n 0}, \epsilon_{n}$ are the initial and final normalized emittances, the factor of the emittance reduction $C=E_{0} / E_{f}$. The method of electron beam cooling allows further reduction of the transverse emittances after damping rings or guns by 1-3 orders of magnitude.

In this paper, we have evaluated the effects of the laser-Compton interaction for transverse cooling using the Monte Carlo code CAIN.2 The simulation calculates the effects of the nonlinear Compton scattering between the laser photons and the electrons during a multi-cooling stage. Next, we examine the optics for cooling with and without chromatic correction. The laser-Compton cooling for JLC/NLd at $E_{0}=2 \mathrm{GeV}$ is considered in section 4. A summary of conclusion is given in section 5 .

${ }^{*}$ This work was supported in part by the U.S. Department of Energy under Contract No. DEAC03-76SF00098. 
Table 1. Parameters of the electron beams for laser-Compton cooling. The value in the parentheses is given by Telnov's formulas.

\begin{tabular}{ccccccc}
\hline$E_{0}(\mathrm{GeV})$ & $E_{f}(\mathrm{GeV})$ & $C$ & $\epsilon_{n, x} / \epsilon_{n, y}(\mathrm{~m} \cdot \mathrm{rad})$ & $\beta_{x} / \beta_{y}(\mathrm{~mm})$ & $\sigma_{z}(\mathrm{~mm})$ & $\delta(\%)$ \\
\hline 2 & 0.2 & 10 & $7.4 \times 10^{-8} / 2.9 \times 10^{-8}$ & $4 / 4$ & 0.5 & $11(9.8)$ \\
5 & 1 & 5 & $3.0 \times 10^{-6} / 3.0 \times 10^{-6}$ & $0.1 / 0.1$ & 0.2 & $19(19)$ \\
\hline
\end{tabular}

Table 2. Parameters of the laser beams for laser-Compton cooling. The value in the parentheses is given by Telnov's formulas.

\begin{tabular}{ccccccc}
\hline$E_{0}(\mathrm{GeV})$ & $\lambda_{L}(\mu \mathrm{m})$ & $x_{0}$ & $A(\mathrm{~J})$ & $\xi$ & $R_{L, x} / R_{L, y}(\mathrm{~mm})$ & $\sigma_{L, z}(\mathrm{~mm})$ \\
\hline 2 & 0.5 & 0.076 & $300(56)$ & $2.1(2.2)$ & $0.3 / 0.3$ & 1.25 \\
5 & 0.5 & 0.19 & $20(4)$ & $1.5(1.5)$ & $0.1 / 0.1$ & 0.4 \\
\hline
\end{tabular}

\section{Laser-Electron Interaction}

\subsection{Laser-Electron Interaction}

In this section, we describe the main parameters for laser-Compton cooling of electron beams. A laser photon of energy $\omega_{L}$ (wavelength $\lambda_{L}$ ) is backward-Compton scattered by an electron beam of energy $E_{0}$ in the interaction point (IP). The kinematics of Compton scattering is characterized by the dimensionless parameter

$$
x_{0} \equiv \frac{4 E_{0} \omega_{L}}{m_{e}^{2} c^{4}}=0.019 \frac{E_{0}[\mathrm{GeV}]}{\lambda_{L}[\mu \mathrm{m}]},
$$

where $m_{e}$ is electron mass. The parameters of the electron and laser beams for laser-Compton cooling are listed in Tables 1 and 2. The parameters of the electron beam with $2 \mathrm{GeV}$ are given for JLC/NLC case in section 4 . The parameters of that with $5 \mathrm{GeV}$ are used for simulation in the next subsection. The wavelength of laser is assumed to be $0.5 \mu \mathrm{m}$. The parameters of $x_{0}$ with the electron energies $2 \mathrm{GeV}$ and $5 \mathrm{GeV}$ are 0.076 and 0.19 , respectively.

The required laser flush energy with $Z_{R} \ll l_{\gamma} \simeq l_{e}$ ist

$$
A=25 \frac{l_{e}[\mathrm{~mm}] \lambda_{\mathrm{L}}[\mu \mathrm{m}]}{E_{0}[\mathrm{GeV}]}(C-1)[\mathrm{J}],
$$

where $Z_{R}, l_{\gamma}\left(\sim 2 \sigma_{L, z}\right)$, and $l_{e}\left(\sim 2 \sigma_{z}\right)$ are the Rayleigh length of laser, and the bunch lengths of laser and electron beams. From this formula, the parameters of $A$ with the electron energies $2 \mathrm{GeV}$ and $5 \mathrm{GeV}$ are $56 \mathrm{~J}$ and $4 \mathrm{~J}$, respectively.

The nonlinear parameter of laser field is

$$
\xi^{2}=4.3 \frac{\lambda_{L}^{2}\left[\mu \mathrm{m}^{2}\right]}{l_{e}[\mathrm{~mm}] E_{0}[\mathrm{GeV}]}(C-1) .
$$

In this study, for the electron energies $2 \mathrm{GeV}$ and $5 \mathrm{GeV}$, the parameters of $\xi$ are 2.2 and 1.5 , respectively.

The rms energy of the electron beam after Compton scattering ist

$$
\sigma_{e}=\frac{1}{C^{2}}\left[\sigma_{e 0}^{2}\left[\mathrm{GeV}^{2}\right]+0.7 x_{0}(1+0.45 \xi)(C-1) E_{0}^{2}\left[\mathrm{GeV}^{2}\right]\right]^{1 / 2}[\mathrm{GeV}],
$$


where the rms energy of the initial beam is $\sigma_{e 0}$ and the ratio of energy spread is defined as $\delta=\sigma_{e} / E_{f}$. If the parameter $\xi$ or $x_{0}$ is larger, the energy spread after Compton scattering is increasing and it is the origin of the emittance growth in the defocusing optics, reacceleration linac, and focusing optics. The energy spreads $\delta$ for the electron energies $2 \mathrm{GeV}$ and $5 \mathrm{GeV}$ are $9.8 \%$ and $19 \%$, respectively.

The equilibrium emittances due to Compton scattering are

$$
\epsilon_{n i, \min }=\frac{7.2 \times 10^{-10} \beta_{i}[\mathrm{~mm}]}{\lambda_{L}[\mu \mathrm{m}]}(i=x, y)[\mathrm{m} \cdot \mathrm{rad}],
$$

where $\beta_{i}$ are the beta functions at IP. From this formula we can see that small beta gives small emittance. However the large change of the beta functions between the magnet and the IP causes the emittance growth. Taking no account of the emittance growth, for the electron energies $2 \mathrm{GeV}$ and $5 \mathrm{GeV}$, the equilibrium emittances are $5.8 \times 10^{-9} \mathrm{~m} \cdot \mathrm{rad}$ and $1.4 \times 10^{-10} \mathrm{~m} \cdot \mathrm{rad}$, respectively. The equilibrium emittances depended on $\xi$ in the case $\xi^{2} \gg 1$ were calculated in Ref. 1.

\subsection{Simulation of Laser-Electron Interaction}

For the simulation of laser-electron interaction, the electron beam is simply assumed to be a round beam in the case of $E_{0}=5 \mathrm{GeV}$ and $C=5$. Taking no account of the emittance growth of optics, the one stage for cooling consists two parts as follows:

1. The laser-Compton interaction between the electron and laser beams.

2. The reacceleration of electrons in linac.

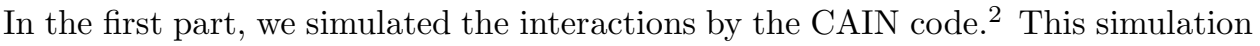
calculates the effects of the nonlinear Compton scattering between the laser photons and the electrons. We assume that the laser pulse interacts with the electron bunch in head-on collisions. The $\beta_{x}$ and $\beta_{y}$ at the IP are fixed to be $0.1 \mathrm{~mm}$. The initial energy spread of the electron beams is $1 \%$. The energy of laser pulse is $20 \mathrm{~J}$. The difference of the pulse energy between the simulation and the formula depends on the transverse sizes of the electron beams at IP. The polarization of the electron and laser beams are $P_{e}=1.0$ and $P_{L}=1.0$ (circular polarization), respectively. When the $x_{0}$ and $\xi$ parameters are small, the spectrum of the scattered photons does not largely depend on the polarization combination. In order to accelerate the electron beams to $5 \mathrm{GeV}$ for recovery of energy in the second part, we simply added the energy $\Delta E=5 \mathrm{GeV}-E_{\text {ave }}$ for reacceleration, where $E_{\text {ave }}$ is the average energy of the scattered electron beams after the laser-Compton interaction.

Here we define the transverse, longitudinal, and 6D emittances in the simulation. The $x, y$-transverse emittance is

$$
\epsilon_{n, i}=\sqrt{\sigma_{i}^{2} \sigma_{i^{\prime}}^{2}-\left(\left\langle i \cdot i^{\prime}\right\rangle-\langle i\rangle\left\langle i^{\prime}\right\rangle\right)^{2}}(i=x, y),
$$

where the symbol \langle\rangle means to take an average of all particles in a bunch. 
The longitudinal emittance is

$$
\epsilon_{n, l}=\sqrt{\sigma_{z}^{2} \sigma_{p_{z}}^{2}-\left(\left\langle z \cdot p_{z}\right\rangle-\langle z\rangle\left\langle p_{z}\right\rangle\right)^{2}} /\left(m_{e} c\right) .
$$

The $6 \mathrm{D}$ emittance is defined as

$$
\epsilon_{6 N}=\epsilon_{n, x} \cdot \epsilon_{n, y} \cdot \epsilon_{n, l} .
$$

Figure 1 shows the longitudinal distribution of the electrons after the first laserCompton scattering. The average energy of the electron beams is $1.0 \mathrm{GeV}$ and the energy spread $\delta$ is 0.19 . The longitudinal distribution seems to be a boomerang. If we assume a short Rayleigh length of laser pulse, the energy loss of head and tail of beams is small. The number of the scattered photons per incoming particle and the average of the photon energy at the first stage are 40 and $96 \mathrm{MeV}$ (rms energy $140 \mathrm{MeV}$ ), respectively.
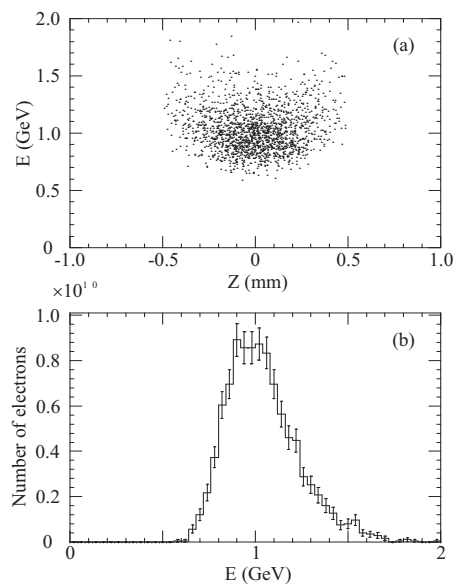

Fig. 1. The longitudinal distribution of the electrons. (a) The energy vs. $z$. (b) The energy distribution of the electrons. The bin size is $40 \mathrm{MeV}$.

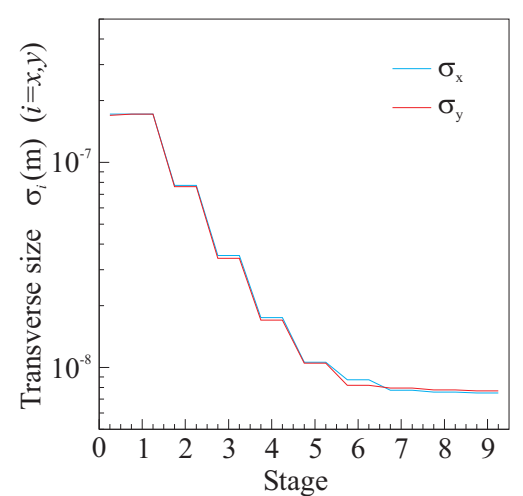

Fig. 2. The transverse sizes of the electron beams.

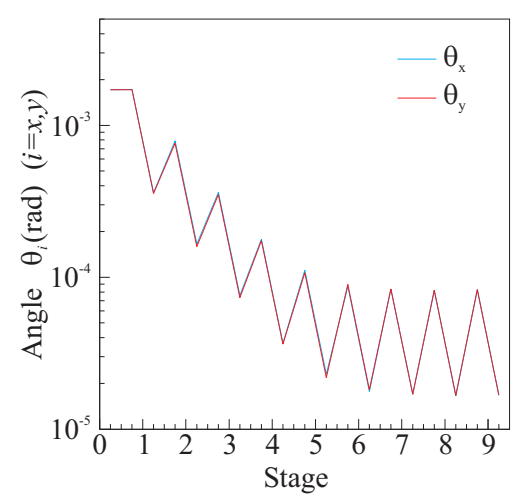

Fig. 3. The angles of the electron beams. 
The transverse sizes of the electron beams in the multi-stage cooling are shown in Fig. 2. During collisions with the laser photons, the transverse distribution of the electrons remains almost unchanged. But they decrease when we focus them for the next laser-Compton interaction due to the lower normalized emittance and the fixed $\beta$-function at IP $\left(\sigma_{i}=\sqrt{\beta_{i} \epsilon_{n, i} / \gamma}\right)$. The angles of the electron beams in the multi-stage cooling are shown in Fig. 3 . As a result of reacceleration, the angles of the electrons decrease. They increase when we focus them for the next laserCompton interaction. Finally the angles attain the average of Compton scattering angle and the effect of cooling saturates.

Figure 1 shows the transverse emittances of the electron beams in the multistage cooling. From Eq.(6),$\epsilon_{n i, \min }=1.4 \times 10^{-10} \mathrm{~m} \cdot \mathrm{rad}$, and the simulation presents $\epsilon_{n i, \min }=1.2 \times 10^{-9} \mathrm{~m} \cdot \mathrm{rad}$. Figure 5 shows the longitudinal emittance of the electron beams in the multi-stage cooling. Due to the increase of the energy spread of the electron beams from $1 \%$ to $19 \%$, the longitudinal emittance rapidly increases at the first stage. After the first stage, the normalized longitudinal emittance is stable. The $6 \mathrm{D}$ emittance of the electron beams in the multi-stage cooling is shown in Fig. 6. The second cooling stage has the largest reduction for cooling. The 8th or 9th cooling stages have small reduction for cooling. The initial and final $6 \mathrm{D}$ emittances $\epsilon_{6 N}$ are $1.5 \times 10^{-13}(\mathrm{~m} \cdot \mathrm{rad})^{3}$ and $1.2 \times 10^{-19}(\mathrm{~m} \cdot \mathrm{rad})^{3}$, respectively.

Figure 7 shows the polarization of the electron beams in the multi-stage cooling. The decrease of the polarization during the first stage is about 0.06. The final polarization $P_{e}$ after the multi-stage cooling is 0.54 .

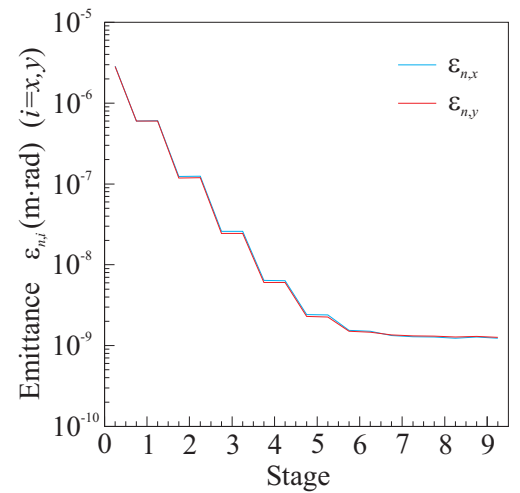

Fig. 4. The transverse emittances of the electron beams.

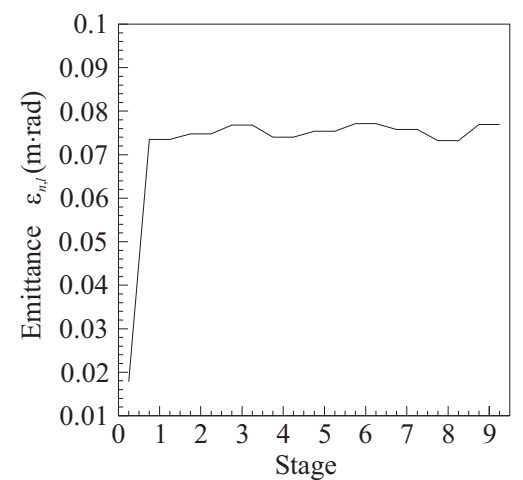

Fig. 5. The longitudinal emittance of the electron beams.

\section{Optics Design for Laser-Compton Cooling}

\subsection{Optics without chromaticity correction}

There are three optical devices for the laser-Compton cooling of electron beams as follows:

1. The focus optics to the first IP. 

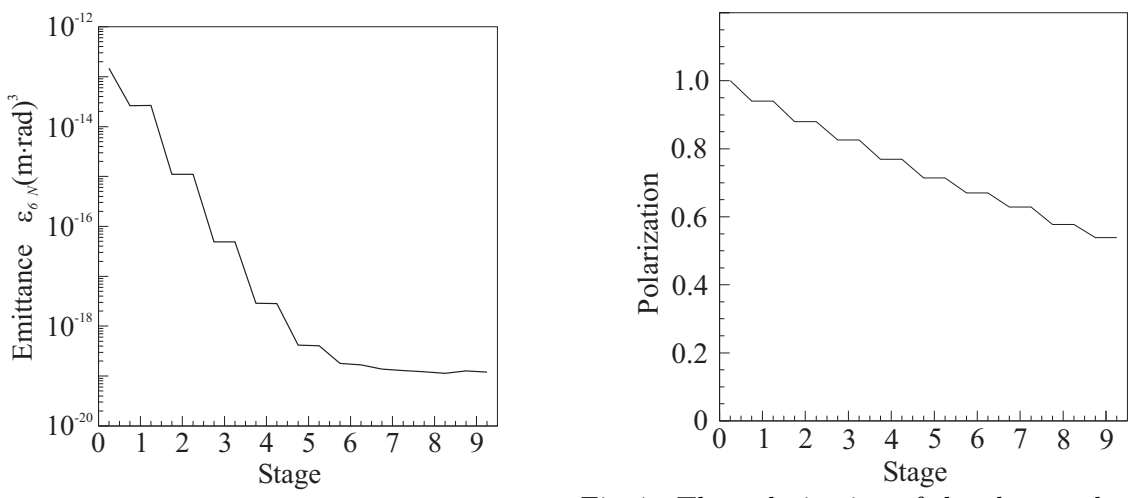

Fig. 6. The $6 \mathrm{D}$ emittance of the electron beams. Fig. 7. The polarization of the electron beams.

2. The defocus optics from the first IP to the reacceleration linac.

3. The focus optics from the linac to the next IP.

Figure 8 shows schematics of the laser-Compton cooling of electron beams. The optics 1 is focusing the electron beams from a few meters of $\beta$-function to several millimeters in order to effectively interact them with the laser beams. The optics 2 is defocusing them from several millimeters to a few meters for reacceleration of electron beams in linac. In a multi-stage cooling system, the optics 3 is needed for cooling in the next stage. The problem for the focus and defocus optical devices is the energy spread of electrons and the electron beams with a large energy spread are necessary to minimize or correct the chromatic aberrations avoiding emittance growth.

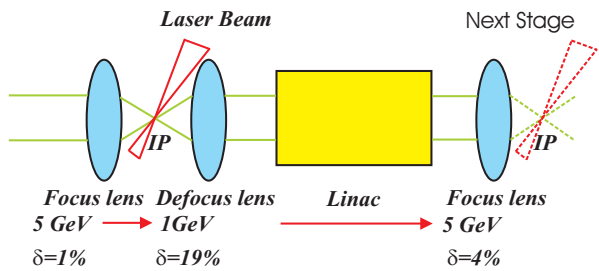

Fig. 8. Schematics of the laser-Compton cooling of electron beams.

In this subsection, we discuss the optics for laser-Compton cooling without chromatic corrections. For the focus and defocus of the beams, we use the final doublet system which is similar to that of the final focus system of the future linear colliders. 3 The pole-tip field of the final quadrupole $B_{T}$ is limited to $1.2 \mathrm{~T}$ and the pole-tip radius $a$ is greater than $3 \mathrm{~mm}$. The strength of the final quadrupole is

$$
\kappa=B_{T} /(a B \rho) \leq 120 / E[\mathrm{GeV}]\left[\mathrm{m}^{-2}\right],
$$

where $B, \rho$, and $E$ are the magnetic field, the radius of curvature and the energy of the electron beams. In our case, the electron energies in the optics 1,2 , and 3 are $5.0,1.0$, and $5.0 \mathrm{GeV}$, respectively and the limit of the strength of the quadrupole 
in laser cooling is much larger than that of the final quadrupole of the future linear colliders. Due to the low energy beams in laser cooling, the synchrotron radiation from quadrupoles and bends is negligible.

The difference of three optical devices is the amount of the energy spread of the beams. In the optics 1,2 , and 3 , the beams have one, several tens, and a few $\%$ energy spread. In order to minimize the chromatic aberrations, we need to shorten the length between the final quadrupole and the IP. In this study, the length from the face of the final quadrupole to the IP, $l$ is assumed to be $2 \mathrm{~cm}$. Here we estimated the emittance growth in the optics 2, because the chromatic effect in the optics 2 is the most serious. Figure 9 shows the defocus optics without chromaticity correction for laser-Compton cooling by the MAD code. The input file is attached to Ref. 5 . The parameters of the electron beam for laser-Compton cooling at $E_{0}=5 \mathrm{GeV}$ and $C=5$ are listed in Table 3. The initial $\beta_{x}$ and $\beta_{y}$ after laser-Compton interaction are $20 \mathrm{~mm}$ and $4 \mathrm{~mm}$, respectively. The final $\beta_{x}$ and $\beta_{y}$ are assumed to be $2 \mathrm{~m}$ and $1 \mathrm{~m}$, respectively. The initial and final $\alpha_{x(y)}$ with no energy spread $\delta=0$ are 0 in this optics. The strength $\kappa$ of the final quadrupole for the beam energy of $1 \mathrm{GeV}$ from Eq. (10) is assumed to be $120 \mathrm{~m}^{-2}$.

Table 3. Parameters of the electron beam for laser-Compton cooling at $E_{0}=5 \mathrm{GeV}$ and $C=5$ for the optics design.

\begin{tabular}{ccccc}
\hline$E_{0}(\mathrm{GeV})$ & $\epsilon_{n, x} / \epsilon_{n, y}(\mathrm{~m} \cdot \mathrm{rad})$ & $\beta_{x} / \beta_{y}(\mathrm{~mm})$ & $\sigma_{x} / \sigma_{y}(\mathrm{~m})$ & $\sigma_{z}(\mathrm{~mm})$ \\
\hline 5 & $1.06 \times 10^{-6} / 1.6 \times 10^{-8}$ & $20 / 4$ & $3.3 \times 10^{-5} / 1.8 \times 10^{-7}$ & 0.2 \\
\hline
\end{tabular}

In our case, the chromatic functions $\xi_{x}$ and $\xi_{y}$ are 18 and 148 , respectively. The momentum dependence of the emittances in the defocus optics without chromaticity correction is shown in Fig. 10. In the paper, the analytical study by thin-lens approximation has been studied for the focusing system, and here the transverse emittances are calculated by a particle-tracking simulation. The 10000 particles are tracked for the transverse and longitudinal Gaussian distribution by the MAD code. The relative energy spread $\delta$ is changed from 0 to 0.4 . Due to the larger chromaticity $\xi_{y}$, the emittance $\epsilon_{y}$ is rapidly increasing with the energy spread $\delta$. If we set a limit of $200 \%$ for $\Delta \epsilon_{i} / \epsilon_{i}(i=x, y)$, the permissible energy spread $\delta_{x}$ and $\delta_{y}$ are 0.11 and 0.012 which mean the momentum band widths $\pm 22 \%$ and $\pm 2.4 \%$, respectively. The results are not sufficient for cooling at $E_{0}=5 \mathrm{GeV}$ and $C=5$, because the beams through the defocusing optics have the energy spread of several tens $\%$. On the one hand, the optics can be useful as the optics 1 and 3 with the energy spread of a few $\%$.

\subsection{Optics with chromaticity correction}

The optics without chromaticity correction for the optics 2 does not work as we saw in the previous subsection. In this subsection we apply the chromaticity correction for the optics 2 . The lattice for cooling is designed referring to the final focus system of the future linear colliders by K. Oide. The final doublet system 


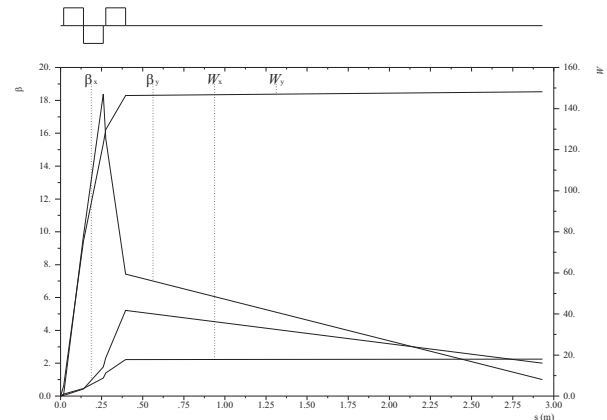

Fig. 9. The defocus optics without chromaticity correction for laser-Compton cooling.
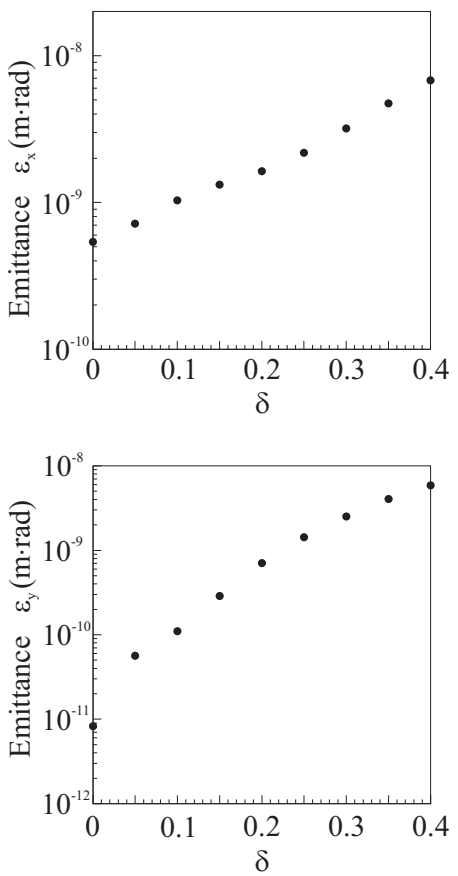

Fig. 10. Momentum dependence of the emittances in the defocus optics without chromaticity correction.

is the same lattice as the optics before subsection. The method of chromaticity correction uses one family of sextupole to correct for vertical chromaticity and moreover we added two weak sextupoles in the lattice to correct for horizontal chromaticity. Figure 11 shows the defocus optics with chromaticity correction for the laser-Compton cooling. The input file is attached to Ref. 5 . The total length of the lattice is about $63 \mathrm{~m}$.

The momentum dependence of the emittances in the defocus optics with chromaticity correction is shown in Fig. 12. The 10000 particles are tracked for the transverse and longitudinal Gaussian distribution by the MAD code. The relative energy spread $\delta$ is changed from 0 to 0.06 with the conservation $\kappa_{2} \theta_{B}$, where $\kappa_{2}$ and $\theta_{B}$ are the strength of the sextupole and the angle of the bending magnet. The initial $\beta_{x}$ and $\beta_{y}$ after laser-Compton interaction are $20 \mathrm{~mm}$ and $4 \mathrm{~mm}$, respectively. The final $\beta_{x}$ and $\beta_{y}$ are assumed to be $2 \mathrm{~m}$ and $1 \mathrm{~m}$, respectively. The initial and final $\alpha_{x}(y)$ with no energy spread $\delta=0$ are 0 in this optics. After the chromaticity correction, the chromaticity functions $\xi_{x}$ and $\xi_{y}$ are 9.3 and 1.6, respectively. If we set a limit of $200 \%$ for $\Delta \epsilon_{i} / \epsilon_{i}(i=x, y)$, the permissible energy spread $\delta_{x}$ and $\delta_{y}$ are 0.040 and 0.023 which mean the momentum band widths $\pm 8 \%$ and $\pm 4.6 \%$, respectively. By the comparison with the results of the optics without chromaticity correction at a limit of $200 \%$ for $\Delta \epsilon_{i} / \epsilon_{i}(i=x, y)$, the $\epsilon_{y}$ of the optics with chromaticity correction is about two times larger than that of the one before subsection, 
but the $\epsilon_{x}$ of the optics with chromaticity correction is three times smaller than that of the one before. The results are still not sufficient for cooling with $E_{0}=5 \mathrm{GeV}$ and $C=5$. These results emphasize the need to pursue further ideas for plasma lens. 8
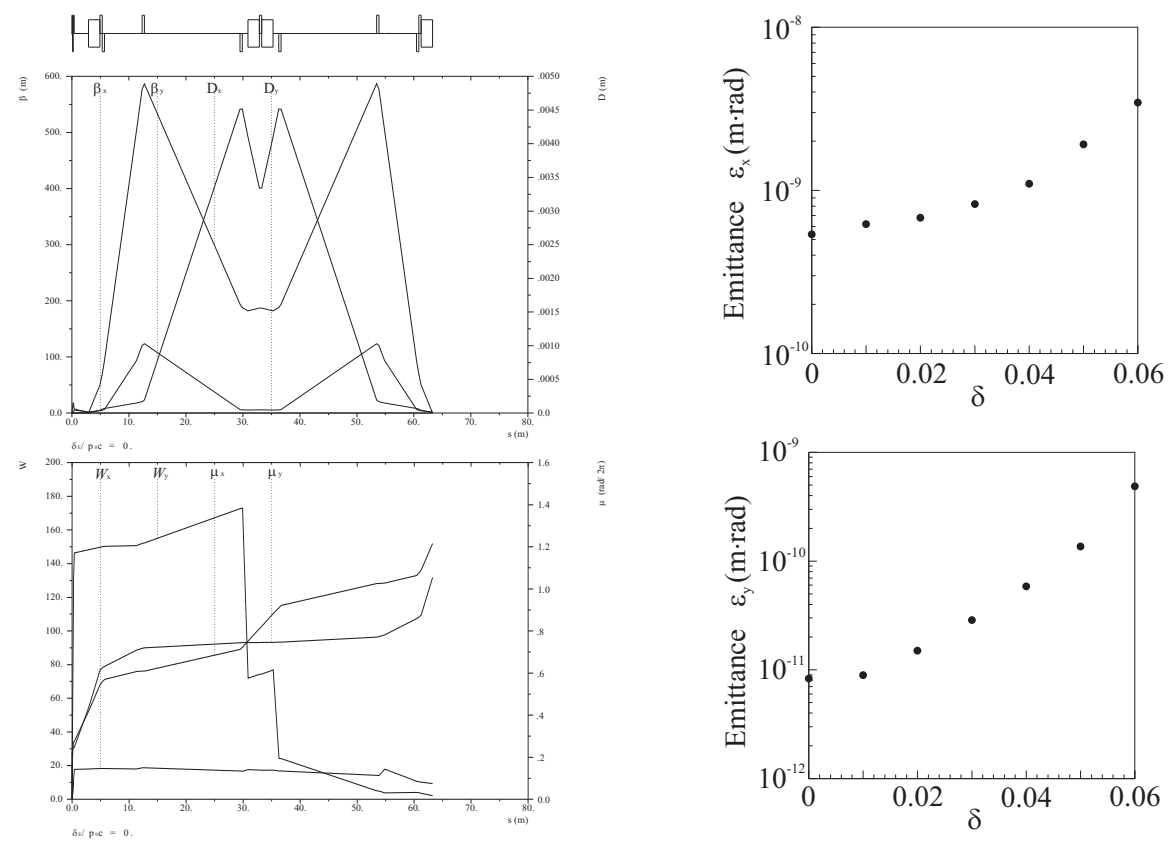

Fig. 11. The defocus optics without chromaticity correction for laser-Compton cooling.

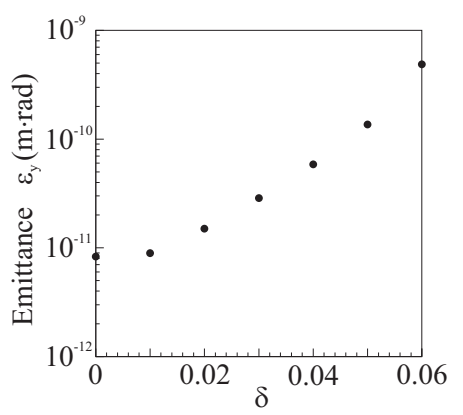

Fig. 12. Momentum dependence of the emittances in the defocus optics with chromaticity correction.

\section{Laser-Compton Cooling for JLC/NLC at $E_{0}=2 \mathrm{GeV}$}

\subsection{Optics}

For the future linear colliders, the method of laser-Compton cooling is effective to reduce the transverse emittances after damping rings. Where can it be placed? There are two possibilities for JLC/NLd 9 as follows:

1. After the first bunch compressor (BC1) and before the pre-linac. $E_{0}=2 \mathrm{GeV}$ and $\sigma_{z}=0.5 \mathrm{~mm}$.

2. After the second bunch compressor (BC2) and before the main linac. $E_{0}=10$ $\mathrm{GeV}$ and $\sigma_{z}=0.1 \mathrm{~mm}$.

Case 2 needs a large energy for recovery after Compton scattering and we consider case 1 in this study. The parameters of the electron and laser beams for laserCompton cooling for JLC/NLC at $E_{0}=2 \mathrm{GeV}$ and $C=10$ are listed in Tables $[1$ and 2. The energy of laser pulse is $300 \mathrm{~J}$. The simulation results of the laser-electron 
interaction by the CAIN code are summarized as follows. The energy spread of the electron beam is $11 \%$. The decrease of the longitudinal polarization of the electron beam is $0.038\left(P_{e}=1.0, P_{L}=1.0\right)$. The number of the scattered photons per incoming particle and the average of the photon energy are 200 and $8.9 \mathrm{MeV}$ (rms energy $19 \mathrm{MeV})$, respectively.

Table 4. Parameters of the defocus optics for laser-Compton cooling for JLC/NLC at $E_{0}=2 \mathrm{GeV}$ and $C=10$.

\begin{tabular}{ccccc}
\hline$l$ & Length of Q1 & Field of Q1 & Aperture & Total length \\
\hline $5 \mathrm{~mm}$ & $2 \mathrm{~cm}$ & 1.2 Tesla & $0.5 \mathrm{~mm}$ & $7.4 \mathrm{~cm}$ \\
\hline
\end{tabular}

The electron energy after Compton scattering in case 2 is $0.2 \mathrm{GeV}$ and the strength of the final quadrupole from Eq. (10) is $600 \mathrm{~m}^{-2}$. Table 1 lists the parameters of the defocusing optics for laser-Compton cooling for JLC/NLC at $E_{0}=2$ $\mathrm{GeV}$ and $C=10$. The final $\beta_{x}$ and $\beta_{y}$ are assumed to be $1 \mathrm{~m}$ and $0.25 \mathrm{~m}$, respectively. The chromaticity functions $\xi_{x}$ and $\xi_{y}$ are 18 and 23, respectively. Using the MAD code, the emittance growth in the defocus optics are

$$
\begin{aligned}
& \Delta \epsilon_{n, x}^{\text {defocus }}=\epsilon_{n, x}-\epsilon_{n, x 0} \sim 1.0 \epsilon_{n, x 0} \sim 7.6 \times 10^{-8}[\mathrm{~m} \cdot \mathrm{rad}], \\
& \Delta \epsilon_{n, y}^{\text {defocus }}=\epsilon_{n, y}-\epsilon_{n, y 0} \sim 1.6 \epsilon_{n, y 0} \sim 4.6 \times 10^{-8}[\mathrm{~m} \cdot \mathrm{rad}],
\end{aligned}
$$

where the normalized emittances before and after the defocus optics are $\epsilon_{n, i 0}$ and $\epsilon_{n, i}(i=x, y)$, respectively. The emittance growth in the other two focus optics are negligible.

\subsection{Reacceleration Linac}

In the reacceleration linac, there are two major sources of the emittance increase as follows:

1. The emittance growth due to the misalignment of the quadrupole magnet and the energy spread.

2. The emittance growth due to the cavity misalignment.

The emittance growth due to these sources in the reacceleration linac (L-band linac) are formulated by K. Yokoya

$$
\begin{gathered}
\Delta \epsilon_{n, x}^{\text {linac }} \sim 3.4 \times 10^{-9}[\mathrm{~m} \cdot \mathrm{rad}] \sim 0.045 \epsilon_{n, x 0}, \\
\Delta \epsilon_{n, y}^{\text {linac }} \sim 3.4 \times 10^{-9}[\mathrm{~m} \cdot \mathrm{rad}] \sim 0.12 \epsilon_{n, y 0} .
\end{gathered}
$$

The final emittance growth and the final emittance with $C=10$ are

$$
\begin{aligned}
\Delta \epsilon_{n, x} & \sim 7.9 \times 10^{-8}[\mathrm{~m} \cdot \mathrm{rad}] \sim 1.1 \epsilon_{n, x 0} \Rightarrow \epsilon_{n, x} \sim 0.21 \epsilon_{n, x 0}, \\
\Delta \epsilon_{n, y} & \sim 4.9 \times 10^{-8}[\mathrm{~m} \cdot \mathrm{rad}] \sim 1.7 \epsilon_{n, y 0} \Rightarrow \epsilon_{n, y} \sim 0.27 \epsilon_{n, y 0} .
\end{aligned}
$$


The total reduction factor of the $4 \mathrm{D}$ transverse emittance of the laser-Compton cooling for JLC/NLC at $E_{0}=2 \mathrm{GeV}$ is about 18. The decrease of the polarization of the electron beam is 0.038 due to the laser-Compton interaction.

\section{Summary}

We have studied the method of laser-Compton cooling of electron beams. The effects of the laser-Compton interaction for cooling have been evaluated by the Monte Carlo simulation. From the simulation in the multi-stage cooling, we presented that the low emittance beams with $\epsilon_{6 N}=1.2 \times 10^{-19}(\mathrm{~m} \cdot \mathrm{rad})^{3}$ can be achieved in our beam parameters. We also examined the optics with and without chromatic correction for cooling, but the optics are not sufficient for cooling due to the large energy spread of the electron beams.

The laser-Compton cooling for $\mathrm{JLC} / \mathrm{NLC}$ at $E_{0}=2 \mathrm{GeV}$ and $C=10$ was considered. The total reduction factor of the $4 \mathrm{D}$ transverse emittance of the laser-

Compton cooling is about 18. The decrease of the polarization of the electron beam is 0.038 due to the laser-Compton interaction.

\section{Acknowledgments}

We would like to thank Y. Nosochkov, K. Oide, T. Takahashi, V. Telnov, M. Xie, and K. Yokoya for useful comments and discussions.

\section{References}

1. V. Telnov, Phys. Rev. Lett. 78, 4757 (1997); ibid. 80, 2747 (1998); in Proceedings of the 15th Advanced ICFA Beam Dynamics Workshop on Quantum Aspects of Beam Physics, Monterey, CA, Jan 4-9, 1998, BUDKERINP-98-33 (1998).

2. P. Chen, T. Ohgaki, A. Spitkovsky, T. Takahashi, and K. Yokoya, Nucl. Instrum. and Methods Phys. Res. A 397, 458 (1997).

3. Zeroth-Order Design Report for the Next Linear Collider, LBNL-PUB-5424, SLACReport-474 (1996); JLC Design Study, KEK-Report-97-1 (1997); Conceptual Design of a $500 \mathrm{GeV}$ Electron Positron Linear Collider with Integrated X-Ray Laser Facility, DESY-97-048, ECFA-97-182 (1997).

4. H. Grote and F.C. Iselin, The MAD Program (Methodical Accelerator Design) Version 8.19: User's Reference Manual, CERN-SL-90-13-AP (1996).

5. T. Ohgaki, LBNL-44380 (1999).

6. B.W. Montague and F. Ruggiero, CLIC-NOTE-37 (1987).

7. K. Oide, Nucl. Instrum. Meth. Phys. Res. A 276, 427 (1989); in Proceedings of the DPF Summer Study on High Energy Physics in the 1990's, Snowmass, CO, Jun 27-Jul 15, 1988, SLAC-PUB-4806 (1988); in Proceedings of the 1st Workshop on the Japan Linear Collider (JLC), Tsukuba, Japan, Oct 24-25, 1989, KEK-Preprint-89-190 (1989).

8. P. Chen, K. Oide, A.M. Sessler, and S.S. Yu, Phys. Rev. Lett. 64, 1231 (1990); in Proceedings of the Fourteenth International Conference on High Energy Accelerators, Tsukuba, Aug 22-26, 1989, SLAC-PUB-5060 (1989).

9. K. Yokoya, in Proceedings of the International Symposium on New Visions in LaserBeam Interactions, Tokyo, Oct 11-15, 1999. 